\title{
Comparison Study between Fraunhofer and Fresnel Diffraction in Case of
}

\section{Coherent and Incoherent Illumination}

\author{
Hassan Hammadi1,*, Ayad A. AL-Ani ${ }^{2}$ \\ ${ }^{1}$ Department of physics, College of science, Al-Nahrain University, Jadriyha 64055, Baghdad, Iraq \\ ${ }^{2}$ Department of Information and Communication Engineering, College of information engineering, Al-Nahrain \\ University, Baghdad, Iraq
}

\begin{tabular}{ll}
\hline \multicolumn{1}{c}{ Articles Information } & Abstract \\
\hline Received: & \\
17.02.2020 & In this paper, the effect of Coherent and Incoherent illumination in case of \\
Accepted: & Fraunhofer and Fresnel diffraction have been studied. The comparison has been \\
20.05 .2020 & studied qualitatively in terms of Point Spread Function "PSF" and Modulation \\
Published: & Transfer Function "MTF". This study assumed that there is no aberration and \\
04.06 .2020 & the medium is homogenous i.e.no turbulence. The results show that imaging in \\
Keywords: & case of Fraunhofer diffraction pattern is better than imaging in case of Fresnel \\
Fraunhofer diffraction & diffraction pattern, and for the two type of illumination. Moreover, imaging \\
Fresnel diffraction & using incoherent illumination is better than imaging using coherent \\
Point Spread Function (PSF) & illumination, and for the two types of diffraction pattern \\
Modulation Transfer Function & \\
Coherent illumination & \\
Incoherent illumination & \\
\hline DOI: $10.22401 /$ ANJS.23.2.06 & \\
* Corresponding author: hasansaw61@gmail.com \\
\hline
\end{tabular}

\section{Introduction}

In optical systems, we often are using two important terminologies. These are: the Point spread function and Modulation Transfer Function. The Point Spread Function "PSF" is defined as the energy distributed on the image plane due to a point source located on the object plane [1]. While, the Modulation Transfer Function is an Essential support in the image forming ability process in optical system. The MTF is calculable via the lens design data, and uses to provide means the expressing the image quality of optical system quantitatively [2]. A diffraction process is defined as the curvature of rays across corners or aperture to reach to the points that located behind the corners. In the diffraction phenomenon a description of every point in the wave front As set of single spherical wavelets. These behaviors are shown when the waves come across a slit or partition that is similar in size to its wavelength. The above of this effect happens when the Electromagnetic waves travel via a medium with specific refractive indexes or when sound waves cross with different acoustic impedance via the medium. All waves, including sound waves, water waves, and electromagnetic waves like recognizable X-rays and radio waves are capable of diffraction[3].

In science, two wave sources are completely coherent if they have a continual difference in phase in almost the same frequency and waveformit is known as coherent illumination.

Coherence is also a perfect wave property that allows for basal interference. if two wave have different phase and in different frequency and different waveform it is known as the incoherent illumination [5].

\section{Theoretical Background}

Diffraction Pattern can be categorized into two types. These types of Diffraction pattern are: Fraunhofer diffraction Pattern and Fresnel diffraction Pattern. Fraunhofer diffraction Pattern deal with the pattern of diffraction is finding at a distance from the object. Fraunhofer diffraction occurred when the criteria in equation (1) is satisfied [7]: 


\title{
Al-Nahrain Journal of Science
}

\author{
ANJS, Vol.23 (2), June, 2020, pp. 39 - 43
}

$$
\frac{W^{2}}{L \lambda} \ll 1
$$

where $\mathrm{W}$ is the size of the slit, $\mathrm{L}$ is the distance between the object and aperture, and $\lambda$ is the wavelength of the incident light [5].

While, the Fresnel diffraction pattern is applied on the waves that located near the lens, i.e.: not on the far away from the lens [4]. Fresnel diffraction pattern used to explain the diffraction pattern that happen from wave's passes through aperture or passes around object in case of object is close to the waves [6]. Fresnel diffraction pattern can be express by the specified parameter named, Fresnel number "F". For the optical system: when F $>1$, the diffraction pattern of the wave is considered as Fraunhofer diffraction pattern, as the near to the object, the equation can be written as [4]: $\frac{F \theta^{2}}{4} \ll 1$. where: $\theta$ is the angle between the wave front and the pupil function [7]. For these two cases we can represent:

i. In case of Fraunhofer diffraction pattern and free aberration, the pupil function $\operatorname{pup}(\boldsymbol{x}, \boldsymbol{v}) "$ is given by [7]:

$$
\operatorname{pup}(k, \quad v)=1
$$

ii. In case of Fresnel diffraction pattern and free aberration, the pupil function is given by [7]:

$$
\operatorname{pup}(k, \quad v)=e^{i \varphi_{\text {Free }}} \quad \ldots \text {... (3) }
$$

where: $\varphi_{-}$Fre is the phase difference due to Fresnel incident wave, and $\varphi_{-}$Fre is given by [7]:

$$
\varphi_{\text {Fre. }}=\left(\text { Fre } \cdot a^{2}\right) \cdot K
$$

where Fre is the degree of sphericity, Fre $=1,2$, $3, \ldots$ and $a$ is the normalized distance from the center of the pupil function, which is given by [8]:

$$
a^{2}=\frac{\left(k-k_{c}\right)^{2}+\left(v-v_{c}\right)^{2}}{R^{2}}
$$

where $\mathrm{K}$ is the wave number $=2 \pi / \lambda$ and $\lambda$ is the incident wavelength. $v$ and $k$ are the spatial coordinates of the pupil function, $\left(v_{c}, k_{c}\right)$ is the center coordinate of the pupil (aperture) function. Now, according to the type of illumination, whether its incoherent illumination or coherent illumination, the point spread function "PSF" of an optical system is given by [1]:

i. For incoherent illumination, the point spread function "PSF $(x, y)$ " is given by [1]:

$$
\operatorname{PSF}(x, y)=|F T(\operatorname{pup}(k, v))|^{2}
$$

ii. For coherent illumination, the point spread function is given by [1]:

$$
\operatorname{PSF}(x, y)=|F T(\operatorname{pup}(k, v))|
$$

The Optical Transfer Function "OTF ", equal the Fourier Transform of the Point Spread Function, represent the response of the optical system in frequency domain, given [1]:

$$
\operatorname{OTF}(u, v)=F T[P S f(x, y)]
$$

The OTF is a complex function, therefore to study the response of the optical system in frequency domain, we evaluate what is called the Modulation Transfer Function "MTF ", equal to the absolute value of the Optical Transfer Function, i.e. [9]:

$$
\operatorname{MTF}(u, v)=|\operatorname{OTF}(u, v)|
$$

where $u$ and $v$ represent the coordinate in frequency domain.

\section{Numeric Results}

The comparison between the two types of diffraction pattern. i.e. Fraunhofer diffraction pattern and Fresnel diffraction pattern has been studied in terms of PSF and MTF, the study have been done for two different radius of aperture: $R=$ 10 pixels (small aperture radius) and $\mathrm{R}=40$ pixels (Large aperture radius). Furthermore, the comparison have been studied for the two types of illumination, i.e. coherent illumination and Incoherent illumination. The results are evaluated by using equations $2,3,6$, and 7 . Figures 1 and 2 represent the one dimensional PSF for Fraunhofer diffraction pattern in case of incoherent illumination and coherent illumination, when the radius of the pupil function are: $R=10$ pixels and 40 pixels, respectively. The axes of the above figures represent: $\mathrm{y}$-axis represent the point spread function and the $\mathrm{x}$-axis represent the spatial coordinate.

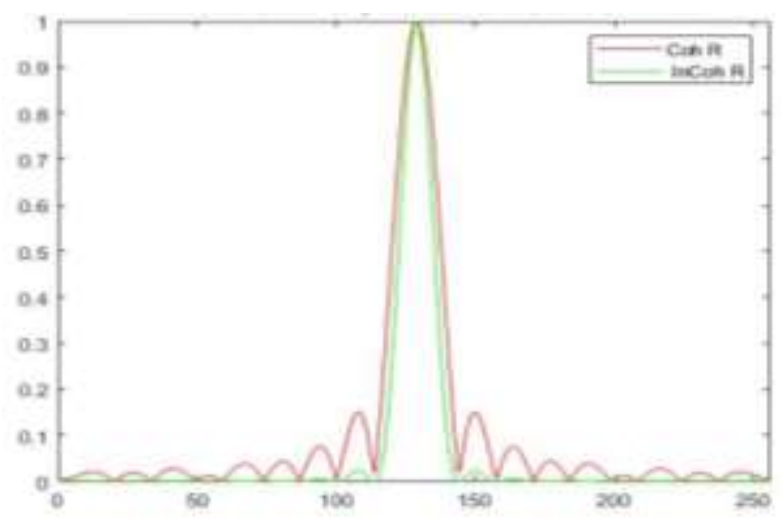

Figure 1. The PSF of the Fraunhofer diffraction pattern in case of incoherent and coherent illumination, when $\mathrm{R}=10$ pixels. 


\section{Al-Nahrain Journal of Science}

ANJS, Vol.23 (2), June, 2020, pp. 39 - 43

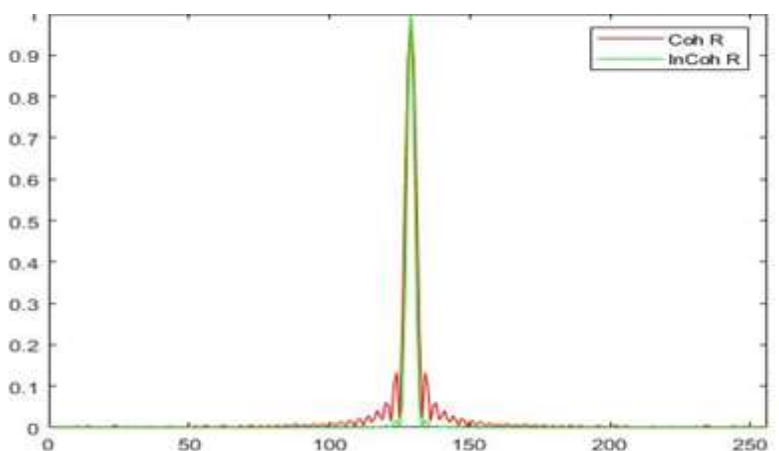

Figure 2. The PSF of the Fraunhofer diffraction pattern in case of incoherent and coherent illumination, when $R=40$ pixels.

Figures 3 and 4 represent the one dimensional Modulation Transfer Function (MTF) for Fraunhofer diffraction pattern in case of incoherent illumination and coherent illumination, when: $\mathrm{R}=10$ pixels and 40 pixels, respectively. The axes of the above figures represent: y-axis represent the modulation transfer function and the $\mathrm{x}$-axis represent the spatial frequency coordinate.

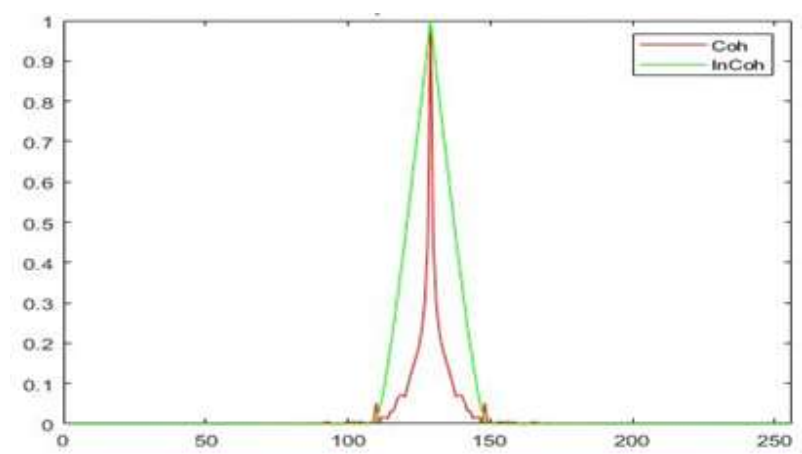

Figure 3. The MTF of the Fraunhofer diffraction pattern in case of incoherent and coherent illumination, when $\mathrm{R}=10$ pixels.

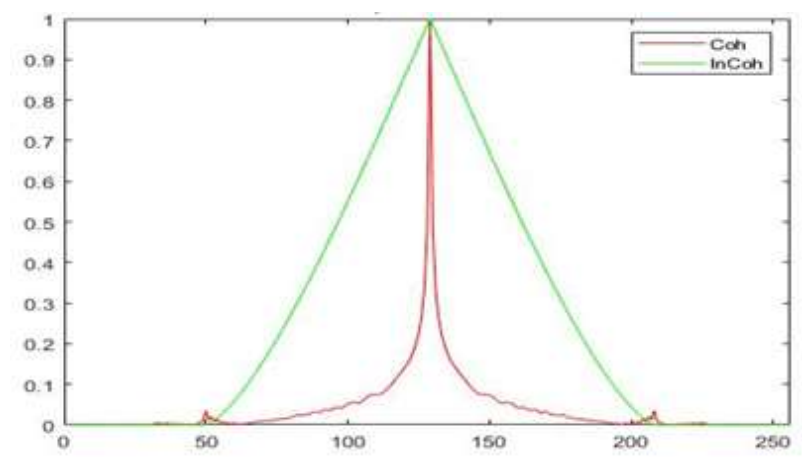

Figure 4. The MTF of the Fraunhofer diffraction pattern in case of incoherent and coherent illumination, when $\mathrm{R}=40$ pixels.
Figures 5 and 6 represent the PSF for Fresnel diffraction pattern in case of incoherent illumination and coherent illumination when: $\mathrm{R}=$ 10 pixels and 40 pixels, respectively.

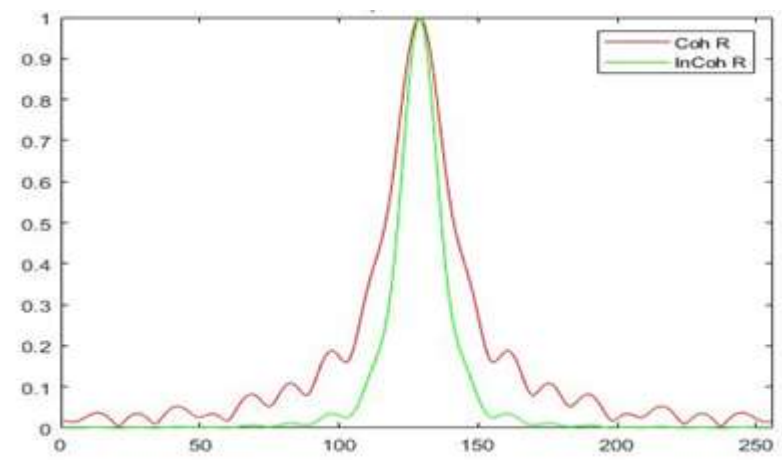

Figure 5. The PSF of the Fresnel diffraction in case of incoherent and coherent when: $R=10$ pixels.

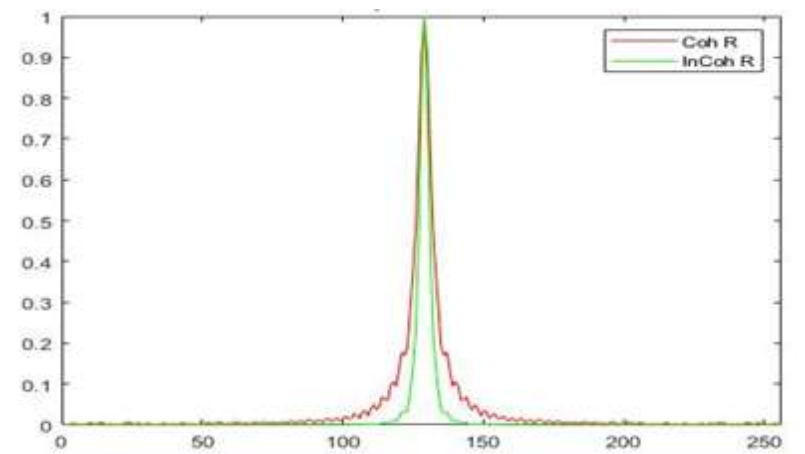

Figure 6. The PSF of the Fresnel diffraction in case of incoherent and coherent when $\mathrm{R}=40$ pixels.

Figures 7 and 8 represent the MTF for Fresnel diffraction in case of incoherent illumination and coherent illumination when: $R=10$ pixels and 40 pixels, respectively. Figures 9 and 10 represent the comparison between MTF Fresnel diffraction pattern and MTF Fraunhofer diffraction pattern, in case of coherent illumination when: $R=10$ pixels and 40 pixels, respectively. Figures 11 and 12 represent the comparison between MTF Fresnel diffraction pattern and MTF Fraunhofer diffraction pattern in case of incoherent illumination when $\mathrm{R}=10$ pixels and 40 pixels, respectively. 


\section{Al-Nahrain Journal of Science}

ANJS, Vol.23 (2), June, 2020, pp. 39 - 43

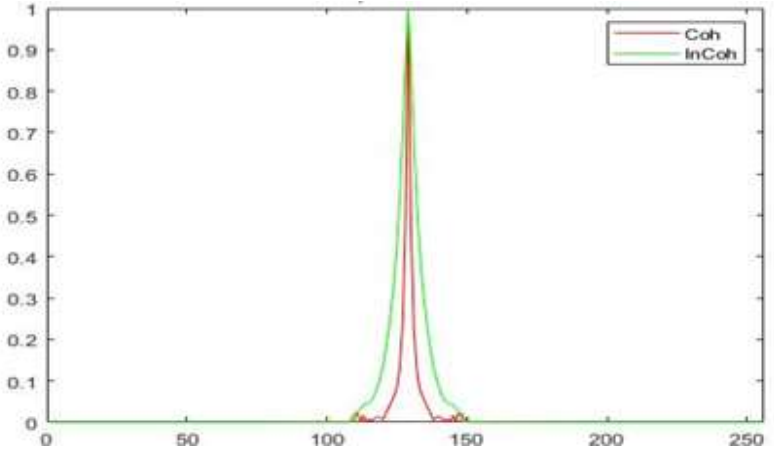

Figure 7. The MTF of the Fresnel diffraction in case of incoherent and coherent when $R=10$ pixels.

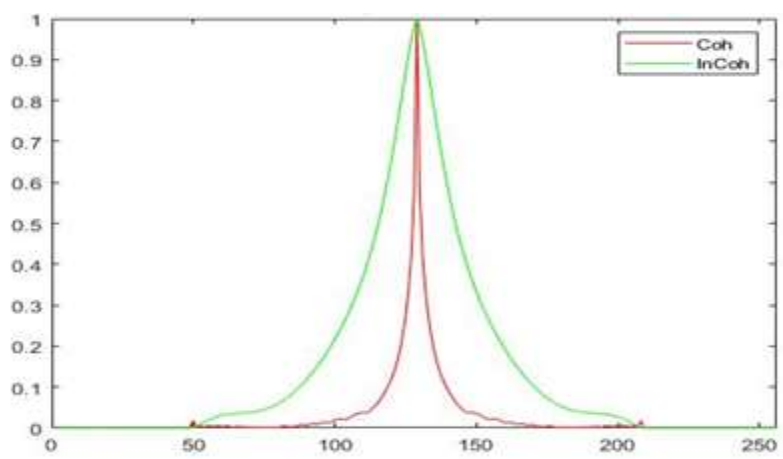

Figure 8. The MTF of the Fresnel diffraction in case of incoherent and coherent when $R=40$ pixels

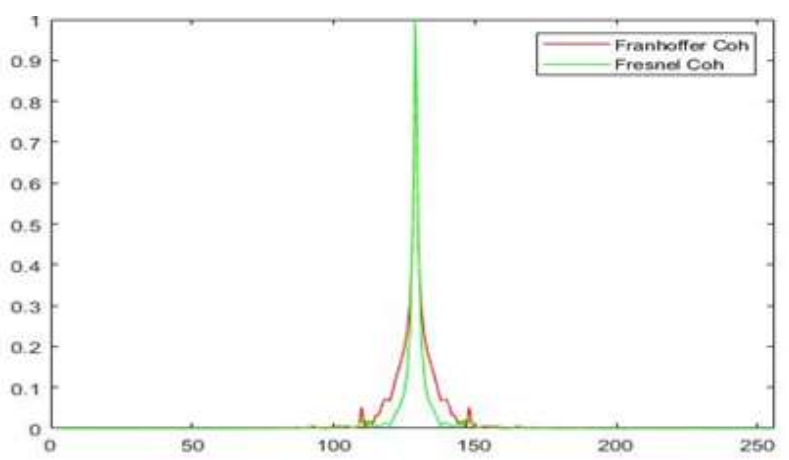

Figure 9. The MTF Fresnel Diffraction Pattern and MTF Fraunhofer diffraction, in case of coherent illumination when $\mathrm{R}=10$ pixels.

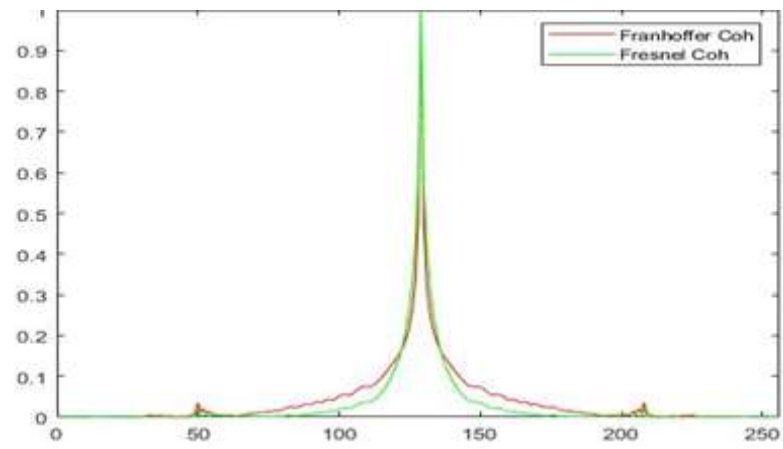

Figure 10. The MTF Fresnel diffraction Pattern and MTF Fraunhofer diffraction Pattern, in case of coherent illumination when $\mathrm{R}=40$ pixels.

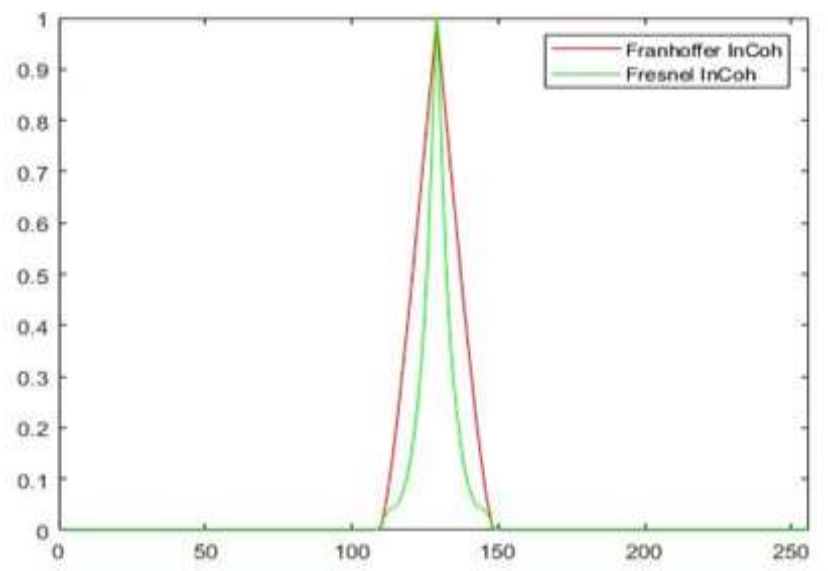

Figure 11. The MTF Fresnel Diffraction Pattern and MTF Fraunhofer diffraction, in case of incoherent illumination, when $\mathrm{R}=10$ pixels.

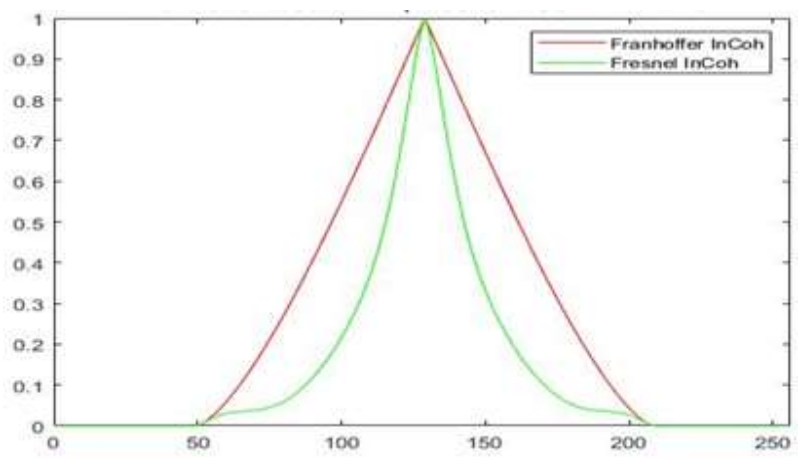

Figure 12. The MTF Fresnel Diffraction Pattern and MTF Fraunhofer diffraction Pattern in case of incoherent illumination, when $\mathrm{R}=40$ pixels. 


\section{Al-Nahrain Journal of Science \\ ANJS, Vol.23 (2), June, 2020, pp. 39 - 43}

\section{Conclusions}

From the above results we can conclude that

1. Imaging through Fraunhofer diffraction pattern is better than imaging through Fresnel diffraction, in case of coherent illumination, especially for large aperture radius.

2. Imaging through Fraunhofer diffraction pattern is better than imaging through Fresnel diffraction, in case of incoherent illumination, especially for large aperture radius.

3. Imaging through incoherent illumination is better than imaging through coherent illumination, and for the two type of diffraction pattern, especially for large aperture radius.

The reasons for the above conclusion belongs to: for incoherent illumination: the PSF treated as intensity function rather than that for coherent illumination which treated as wavefront function. Also, in case of Fraunhofer diffraction, the incident wave front is plane, which means that the phase difference is negligible while for Fresnel diffraction, the incident wave front is spherical, which means that the phase difference is not negligible.

\section{References}

[1] Sheer, A. H.; Al-Ani, A. A.; "The Effect of Regularization Parameter within non - Blind Restoration Algorithm using Modified Iterative wiener Filter For Medical Images", 1st Annual International Conference for Information and Science "AICIS", Fallujah University, IEEE recorded No. 46152, 19-21 Nov. 2018.

[2] Dereniak, E.; "Optical Design", Lecture note, University of Arizona, Tucson, AZ, 2010.

[3] Rappaport, T.; "Principles and Practice", Prentice Hall communications engineering and emerging technologies series, Prentice Hall, 126-128, 2002.

[4] Kalamata, B.; "Introduction to Fourier optics", Vol. 29, pp. 736-738, (2004)

[5] Emil, W.; "World Scientific Publishing" textbooks of optics, United States, 2001.

[6] Joseph, W. G.; "Introduction to Fourier optics", New York, 1996.

[7] Affleck, B.; "Principal of optics", Born and Wolf, Pergamum, London, 1970.

[8] Al-Ani, A. A.; Al-Ani, L. A.; Ali, S. M.; "Entropy as a Measure of Degradation Effects", J. Saddam Univ. 2(1), 113-136, 1998.

[9] Al-Ani, A. A.; Al-Ani, L. A.; Ali, S. M.; "Quantitative and Qualitative Comparison between Short and Long Exposure Imaging", J. Saddam Univ. 2(1), 83-111, 1998. 